\title{
Comparison of hydrophobic and hydrophilic encapsulation using liposomes prepared from milk fat globule-derived phospholipids and soya phospholipids
}

\author{
Abby K. ThOmpson*, Anne Couchoud, Harjinder SINGH** \\ Riddet Institute, Massey University, Palmerston North, New Zealand
}

Received 3rd June 2008 - Accepted 14 November 2008

\begin{abstract}
Liposomes prepared from a milk fat globule membrane (MFGM) phospholipid fraction have been shown to have significantly different physical and chemical characteristics and appeared to be more stable in a variety of conditions than liposomes prepared from soya phospholipid material. These liposome systems were used to try to encapsulate model hydrophobic ( $\beta$-carotene) and hydrophilic (potassium chromate) compounds. Liposomes produced from the MFGM-derived phospholipids showed significantly higher entrapment efficiencies for both $\beta$-carotene and potassium chromate. The differences were particularly apparent when using the hydrophobic molecules at low ratios of $\beta$-carotene to phospholipid. It is likely that the improved incorporation efficiency for $\beta$-carotene is due to the partitioning of the molecule between the aqueous phase and the phospholipid membrane, a property which will be dependent on the specific composition of the phospholipid material used. The higher encapsulation efficiency for the potassium chromate appeared to reflect the slightly larger diameter of the liposomes produced from the MFGM material. These results suggest that there may be inherent advantages in the use of liposomes prepared from MFGM-derived phospholipids via microfluidization for the encapsulation of both hydrophobic and hydrophilic compounds.
\end{abstract}

\section{encapsulation / liposome / milk fat globule membrane / phospholipid}

\begin{abstract}
摘要 - 源于壬脂肪且膜磷脂和从豆磷脂脂质膛的嗜水和亲水败卵作用的比较。分别由乳 脂肪球膜磷脂和大豆磷脂制成的脂质体的物理化学性质明显不同, 在不同条件下前者的稳定 性优于后者。这两种脂质体用于包埋疏水 ( $\beta$-胡萝卜素) 和亲水( 铬酸钾) 性化合物。源于 脂肪球膜磷脂的脂质体对 $\beta$-胡萝卜素和铬酸钾的捕集效率较高。当疏水性分子 $\beta$-胡萝卜素 与磷脂比例较低时这种差别特别显著。对 $\beta$-胡萝卜素结合效率的改变可能是由于水相和磷 脂膜之间存在分子隔膜, 这种特性取决于所用磷脂材料的特定组成。源于乳脂肪球膜材料 的脂质体对铬酸钾较高的包埋效率可能是由于这种脂质体的直径略大一些。试验结果表明, 通过微射流方法制备的乳脂肪球膜磷脂脂质体可以有效地包埋疏水和亲永化合物。
\end{abstract}

包埋 / 脂质体 / 乳脂肪球膜 / 磷脂

\footnotetext{
* Current address: Department of Food Biosciences, University of Reading, Reading RG6 6AP, UK. **Corresponding author (通讯作者): h.singh@massey.ac.nz
} 


\begin{abstract}
Résumé - Comparaison de l'encapsulation de composés hydrophobes et hydrophiles dans des liposomes préparés à partir de phospholipides provenant soit de globules gras de lait, soit de soja. Il a été montré que les liposomes préparés à partir de la fraction phospholipidique de la membrane de globule gras du lait (MGGL) ont des caractéristiques physiques et chimiques significativement différentes et s'avèrent être plus stables dans nombre de conditions que les liposomes préparés à partir de phospholipides de soja. Ces liposomes ont été utilisés pour tester l'encapsulation de composés modèles hydrophobes ( $\beta$-carotène) et hydrophiles (chromate de potassium). Les liposomes produits à partir de phospholipides MGGL ont montré des efficacités de piégeage significativement plus élevées que ce soit pour le $\beta$-carotène ou pour le chromate de potassium. Les différences étaient particulièrement visibles quand les molécules hydrophobes étaient utilisées à des taux $\beta$-carotène/phospholipide faibles. Il est probable que l'efficacité d'incorporation améliorée pour le $\beta$-carotène soit due au partage de la molécule entre la phase aqueuse et la membrane phospholipidique, propriété dépendante de la composition spécifique du matériel phospholipidique utilisé. L'efficacité d'encapsulation plus élevée pour le chromate de potassium paraissait refléter le diamètre légèrement plus large des liposomes produits à partir de MGGL. Ces résultats suggèrent qu'il peut y avoir des avantages inhérents à l'utilisation de liposomes préparés à partir de phospholipides provenant de MGGL via la microfluidisation pour l'encapsulation de composés hydrophobes comme de composés hydrophiles.
\end{abstract}

encapsulation / liposome / membrane de globule gras / phospholipide

\section{INTRODUCTION}

Milk fat globule membrane (MFGM) phospholipids have been shown to have a number of health benefits $[4,5,8,9,15]$, and MFGM material may be successfully used to stabilize emulsions [3, 20, 21, 32] and produce liposomes [29].

Liposomes are phospholipid-bilayer structures with an aqueous core [33], and are usually produced from highly purified phospholipids extracted from soya oil or egg yolk. The first stage in liposome production is the self-assembly of a bilayer of phospholipid molecules. During the formation of this bilayer, hydrophobic compounds may be incorporated inside the membrane. The ends of this bilayer may then wrap around and seal together to form a liposome, enclosing a portion of the aqueous environment inside the vesicle and thus entrapping any hydrophilic compounds present in the aqueous phase.

The effectiveness of a liposome population at entrapping compounds during vesicle formation is commonly reported as entrapment efficiency. This is the proportion of the desired compound present in the system that is held within the liposome structure, either in the aqueous compartments or incorporated in the bilayer membrane. The calculation of the entrapment efficiency is usually based on the removal of unincorporated material and the assumption that the remaining material is all entrapped. This requires a method to separate free and entrapped species, and is usually based on either size and/or density differences [17].

The bilayer membrane is held together by non-covalent interactions, allowing it to carry a wide variety of hydrophobic compounds. The inclusion of such compounds at up to approximately $10 \mathrm{wt}$. \% will not usually disrupt the membrane, although membrane fluidity and permeability may be affected [14]. For highly hydrophobic materials and liposome preparation methods using organic solvents, entrapment is usually close to $100 \%$ regardless of the liposome size, type and composition. Overall entrapment is thus determined by the total amount of phospholipid present [31]. For molecules of lower hydrophobicity, the location of the compound within the liposome and its entrapment efficiency will depend on its partitioning between the hydrophilic and hydrophobic phases. 
The dehydration-rehydration method used by one research group resulted in entrapment efficiencies ranging from 1 to $34 \%$ for proteins of varying hydrophobicities [16].

Hydrophilic entrapment for a given liposome system is proportional to the entrapped volume of the liposomes [31] and the phospholipid concentration. The use of high concentrations of the hydrophilic material increases the active material to phospholipid ratio, making more efficient use of the phospholipid available. If liposomes were tightly-packed solid spheres in an aqueous environment, the theoretical maximum for entrapment efficiency of the aqueous phase would be $70 \%$, as $30 \%$ of the aqueous phase would be required to fill the gaps between the spheres [12]. However, there is evidence that the bilayer membrane is quite flexible and that liposomes are often non-spherical [1,23], which at very high phospholipid concentrations may allow entrapment efficiencies higher than this theoretical maximum. Reported entrapment efficiencies for hydrophilic materials vary greatly depending on the details of the preparation method and specific hydrophilic compound. The standard thin-film liposome preparation method usually gives a hydrophilic entrapment efficiency of only $1-9 \%$, but repeated freezing and thawing of the solution can increase the efficiency to 35-88\%. Mayhew et al. [13] used a Microfluidizer ${ }^{\circledR}$ to encapsulate cytosine arabinoside, and obtained entrapment efficiencies ranging from 5$75 \%$ depending on the operating conditions and phospholipid concentration.

Previous work in our laboratory has compared the general structure and properties of liposomes produced from MFGM-derived phospholipids using three different techniques - microfluidization, the traditional thin-film hydration and the heating method [28]. These results showed that microfluidization produced liposomes with the smallest overall size and narrowest particle size distribution. This technique has therefore been used for the manufacture of liposomes in subsequent work. We have also found that the unique composition of the MFGMderived phospholipid fractions produces liposomes which have a higher phase transition temperature, thicker membrane and lower membrane permeability when compared with liposomes produced from non-hydrogenated soya phospholipids [27]. The MFGM liposomes also demonstrated an improved stability in a range of $\mathrm{pH}$ conditions, at a variety of storage and processing temperatures, and in the presence of mono- and divalent cations [24]. This suggests that liposomes produced from the MFGM-phospholipid material may provide some advantages in the entrapment of bioactive compounds.

To characterise and compare the entrapment profiles of the liposome dispersions, model hydrophobic and hydrophilic compounds were used. $\beta$-Carotene was chosen as a model hydrophobic compound, as its bright orange colour allowed the presence of the molecule to be followed qualitatively by eye and quantitatively by spectrophotometric techniques. Potassium chromate was chosen as a model hydrophilic compound, as its entrapment and release from liposomes has been shown to be similar to that of other molecules with slow rates of membrane diffusion [19].

\section{MATERIALS AND METHODS}

\subsection{Materials}

Both phospholipid products used in this study are commercially available, and were stored vacuum-packed at $-18{ }^{\circ} \mathrm{C}$. The MFGM-derived phospholipid fraction (Phospholac 600) was provided by the Fonterra Co-operative Group Ltd (New Zealand), and a purified soya phospholipid fraction (Sigma catalogue \#P3644, minimum 30\% phosphatidyl 
choline) was obtained from Sigma-Aldrich (St. Louis, MO, USA). These fractions have been comprehensively characterized in earlier publications [26-29], and contained similar overall levels of phospholipids (72-74\% polar lipids), low levels of nitrogen (1.1-2.4\%) and negligible amounts of triglycerides or cholesterol. The primary difference between the fractions was the distribution of phospholipid classes, with the MFGM-derived fraction containing approximately equal amounts of phosphatidyl choline, phosphatidyl ethanolamine and sphingomyelin; while the soya fraction contained twice the amount of phosphatidyl choline and had no sphingomyelin. The fatty acids in the MFGM-derived phospholipids were also more highly saturated than those of soya origin. All chemicals used were of analytical grade and obtained from Sigma-Aldrich.

\subsection{Determination of liposome formation and structure}

Previous publications confirmed the formation of liposomes from the same microfluidisation technique and phospholipid fractions used here. The techniques used included particle size analysis (as outlined below) and various forms of microscopy to provide images of the liposomes (negative staining transmission electron microscopy, thin section transmission electron microscopy, cryo-field emission scanning electron microscopy and atomic force microscopy [28, 29]).

Negative staining transmission electron microscopy was carried out on samples of liposome dispersions with entrapped $\beta$-carotene and potassium chromate across the range of concentrations used. The liposome dispersions were diluted approximately 1:10 with distilled water. One drop of the diluted sample was combined with a drop of $2 \%$ ammonium molybdate and left for $3 \mathrm{~min}$. The solution was then placed on a copper mesh for $5 \mathrm{~min}$ before the excess liquid was drawn off with filter paper. The mesh was examined using a Philips 201C transmission electron microscope (Eindhoven, The Netherlands).

\subsection{Determination of size of liposomes}

The average hydrodynamic diameter of the liposome dispersions was measured on a Zetasizer 4 (Malvern Instruments Ltd., Worcestershire, UK) using photon correlation spectrometry (PCS) as previously described [27-29]. Each sample was analyzed 3 times at $25{ }^{\circ} \mathrm{C}$ with a sampling time of $99 \mathrm{~s}$ and a scattering angle of $90^{\circ}$. A medium viscosity of $1.054 \mathrm{cP}$ and a refractive index of 1.34 were used for the aqueous phase, with a typical liposome refractive index of 1.45 .

\subsection{Preparation of liposomes containing $\beta$-carotene}

A $95 \% \quad \beta$-carotene powder was obtained from Sigma Aldrich (Sigma catalogue \#C9750). Because pure $\beta$-carotene is insoluble in water, some form of solvent must be used to combine the carotene and the phospholipid. In the literature, pure $\beta$-carotene is usually dispersed in chloroform and the liposome produced using the thin-film method. A variation on this technique was developed as outlined below. Because chloroform is not suitable for incorporation in food systems, experiments were also conducted using ethanol as a food-grade alternative.

The $\beta$-carotene was added to a beaker containing the required amount of the MFGM- or soya-derived phospholipid and either ethanol or chloroform. This mixture was heated to approximately $60{ }^{\circ} \mathrm{C}$ on a hot plate with constant stirring, 
and held until the phospholipid formed a melt. The solvent was then evaporated over an $80{ }^{\circ} \mathrm{C}$ water bath. The aqueous phase was then added (imidazole buffer - $20 \mathrm{mmol} \cdot \mathrm{L}^{-1}$ imidazole, $50 \mathrm{mmmol} \cdot \mathrm{L}^{-1}$ sodium chloride, and $0.02 \%$ sodium azide in Milli Q water, adjusted to $\mathrm{pH} \mathrm{7,} \mathrm{with}$ $1 \mathrm{~mol} \cdot \mathrm{L}^{-1}$ hydrochloric acid) and stirred using a magnetic stirrer until the lipid film had hydrated. The dispersions were cycled through a M-110Y Microfluidizer ${ }^{\circledR}$ (Microfluidics International Corp., MANewton, USA) with a $75 \mu \mathrm{m}$ F12Y-type interaction chamber 5 times at $\sim 103 \mathrm{MPa}$ (17000 psi).

\subsection{Separation of unentrapped $\beta$-carotene}

The hydrophobic nature of the $\beta$-carotene caused the unentrapped material to form aggregates of varying sizes. To remove these aggregates, $3 \mathrm{~mL}$ aliquots of the liposome dispersions were added to $6 \mathrm{~mL}$ of buffer solution and centrifuged at $4000 \times g$ for $4 \mathrm{~h}$ in a CentraMP4R centrifuge (International Equipment Company, MA, USA). The supernatant was removed, and the centrifugation step repeated once more. Centrifugation at this speed removed large $\beta$-carotene aggregates, but did not cause significant sedimentation of the liposomes (as measured by determining the phospholipid concentration of the pellet) or of the very small $\beta$-carotene aggregates. As an additional precaution to check for the loss of liposomes, the particle size distribution of liposome dispersions without any entrapped $\beta$-carotene was compared before and after this centrifugation process, and did not show any changes. To remove the smaller $\beta$-carotene particulates, the supernatant was then passed through a Millex GS $0.45 \mu \mathrm{m}$ filter unit (Millipore, MA, USA). A reddish colour indicating the presence of $\beta$-carotene could be seen on the upstream side of the filter. While very large liposomes may not pass through the filter, the vast majority of vesicles were smaller than $450 \mathrm{~nm}$ and it is unlikely that the exclusion of such a small number of liposome would significantly affect the result.

\section{6. $\beta$-Carotene analysis}

The presence of $\beta$-carotene can be determined qualitatively by eye, however quantitative measurements were required to ensure valid comparisons between systems. The concentration of $\beta$-carotene in a sample was measured quantitatively using a Waters 2690 HPLC Separations Module and an Alltech Prevail C18 Column (150× $4.6 \mathrm{~mm}, 5 \mu \mathrm{m})$.

Samples were prepared for HPLC by adding $1 \mathrm{~mL}$ chloroform to between $0.1-$ $1 \mathrm{~mL}$ of the liposome dispersion, then making up to $5 \mathrm{~mL}$ with methanol. This solubilized both the $\beta$-carotene and the phospholipids, producing a clear solution which was passed through $0.22 \mu \mathrm{m}$ filters before filling into HPLC vials. The mobile phase was 90:10 methanol: chloroform, and absorbance measured at $436 \mathrm{~nm}$. The primary $\beta$-carotene peak eluted at between 8.6-8.8 min, although there was a small peak at $\sim 6$ min. Control samples containing liposome with no $\beta$-carotene present did not produce any peaks under these conditions, and it is likely that the small peak was either due to degradation products of the $\beta$-carotene or to other compounds present in the $95 \% \beta$-carotene powder.

\subsection{Preparation of liposomes containing potassium chromate}

To mimic liposomes with entrapped hydrophilic compounds, the phospholipid material was dispersed in imidazole buffer 
containing $28 \mathrm{mg} \cdot \mathrm{mL}^{-1}$ potassium chromate (adjusted to $\mathrm{pH} 7$ ). Dispersions containing 1,5 , or $10 \%$ phospholipid (wt/wt) were prepared in this buffer and mixed thoroughly using an Ultra-Turrax (JKA, Staufen, Germany). These were then cycled 5 times through a 75- $\mu$ m F12Y-type interaction chamber (M-110Y Microfluidizer, Microfluidics International Corp., Newton, MA, USA) at $103 \mathrm{MPa}$.

To allow comparison between the liposomes containing entrapped material and empty liposomes, liposome dispersions were also prepared using the same technique and phospholipid concentrations in imidazole buffer without any potassium chromate.

\subsection{Separation of unentrapped potassium chromate}

The unentrapped potassium chromate was separated from liposomes containing the entrapped molecules using gel permeation chromatography. Two $\mathrm{mL}$ aliquots of the phospholipid liposome dispersions or potassium chromate solutions were passed through a $10 \times 150 \mathrm{~mm}$ column of Bio Gel P-6 DG desalting gel (BioRad, catalogue 150-0738), using gravity feed and imidazole buffer as the mobile phase. A combination of spectrometry and particle size analysis was used to identify the fractions containing liposomes and those containing unentrapped potassium chromate. It took approximately $6 \mathrm{~min}$ for the elution of the liposomes (both empty liposomes and those containing entrapped potassium chromate) and $27 \mathrm{~min}$ for the free or unentrapped potassium chromate. Samples containing potassium chromate and no phospholipids, potassium chromate and phospholipids not made into liposomes, and potassium chromate with empty liposomes were compared across a range of concentrations, and the potassium chromate peak was the same size and eluted at the same time in all samples of a particular concentration, confirming no interaction between the potassium chromate and the phospholipids. There was a consistent gap of at least $15 \mathrm{~min}$ between the elution of the liposomes and the unentrapped material. The fractions eluting from the column were collected manually using consecutive 2-min intervals for $36 \mathrm{~min}$.

Passing a liposome dispersion through the column separated it into fractions of varying particle sizes, but combining all the fractions containing liposomes resulted in a single sample that had the same average diameter as the original dispersion.

\subsection{Analysis of potassium chromate and liposomes}

The absorbance of the original dispersions and of the fractions eluted from the column was measured at $504 \mathrm{~nm}$. This wavelength corresponds to the peak absorbance for potassium chromate, and a calibration curve for free potassium chromate in imidiazole buffer at the concentrations used during these experiments had an $\mathrm{R}^{2}$-value of 0.998 . Liposome dispersions also have a linear relationship between concentration and absorbance at this wavelength, permitting the absorbance of all samples to be measured at $504 \mathrm{~nm}$ to allow direct comparison and manipulation of the results.

\section{RESULTS AND DISCUSSION}

\subsection{Determination of liposome formation, structure and size}

The liposomes formed in the presence of either $\beta$-carotene or potassium chloride looked identical to empty liposomes in terms of structure, bilayer number and arrangement, and relative size distribution in the negative staining transmission electron microscopy images, confirming that 
Table I. Effect of phospholipid concentration on the mean diameter $\left(\mathrm{Z}_{\text {ave }}\right)$ of liposomes produced from the MFGM- and soya-derived phospholipids via microfluidization.

\begin{tabular}{lcc}
\hline $\begin{array}{l}\text { Phospholipid } \\
\text { concentration }(\% \mathrm{w} / \mathrm{w})\end{array}$ & $\begin{array}{c}\text { MFGM-derived phospholipids } \\
\mathrm{Z}_{\text {ave }}(\mathrm{nm})\end{array}$ & $\begin{array}{c}\text { Soya-derived phospholipids } \\
\mathrm{Z}_{\text {ave }}(\mathrm{nm})\end{array}$ \\
\hline 1 & $97 \pm 6$ & $80 \pm 5$ \\
5 & $99 \pm 3$ & $85 \pm 4$ \\
10 & $110 \pm 5$ & $98 \pm 4$ \\
\hline
\end{tabular}

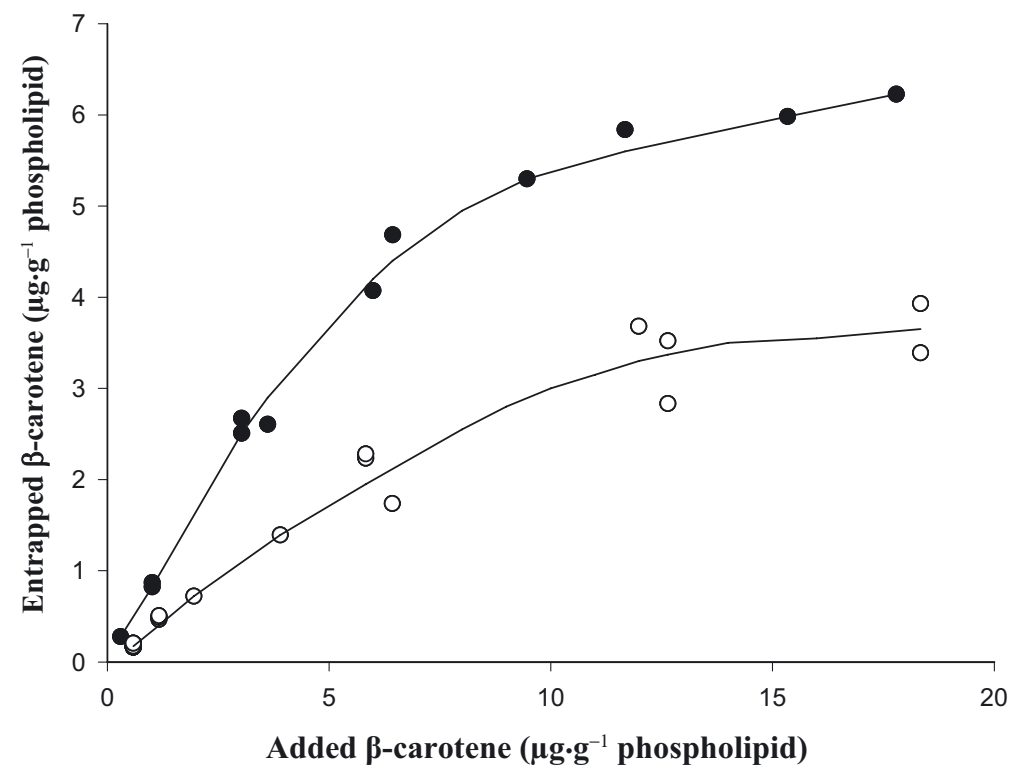

Figure 1. Entrapment of added $\beta$-carotene in liposome membranes using chloroform to disperse the $\beta$-carotene and phospholipid prior to microfluidization. MFGM liposome dispersions $\bullet$, soya liposome dispersions $\bigcirc$.

the addition of the hydrophobic or hydrophilic material had not significantly altered the overall structure of the liposomes.

The liposomes containing entrapped potassium chloride had similar particle size distributions to liposomes prepared solely from the phospholipid fractions (Tab. I). While the liposomes containing $\beta$-carotene were approximately $10 \%$ larger than those without the added hydrophobic molecules, neither the particle size nor the lamellarity of liposomes affects their entrapment efficiency $[30,31]$.

\section{2. $\beta$-Carotene entrapment}

Figure 1 shows the total amount of $\beta$-carotene entrapped per gram of phospholipid (using chloroform) as a function of the added $\beta$-carotene per gram of phospholipid. As expected, the amount of $\beta$-carotene entrapped increased almost linearly with increasing $\beta$-carotene addition up to $\sim 6 \mu \mathrm{g}$ per gram of phospholipid. However, upon addition of $>6 \mu \mathrm{g} \beta$-carotene per gram of phospholipid the amount of entrapped $\beta$-carotene 


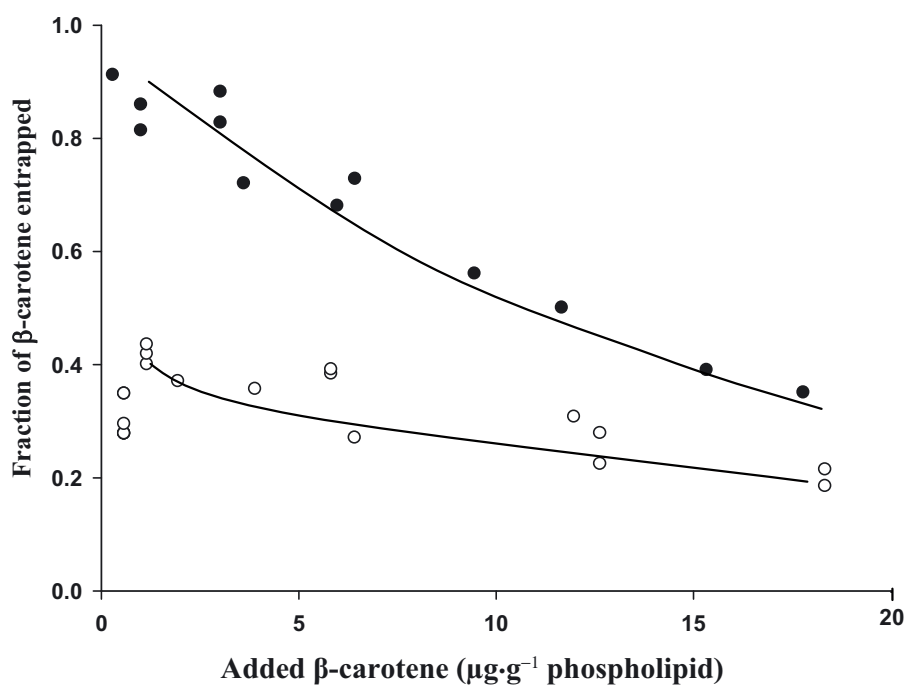

Figure 2. Entrapment efficiency for added $\beta$-carotene in liposome membranes using chloroform to disperse the $\beta$-carotene and phospholipid prior to microfluidization. MFGM liposome dispersions $\bullet$, soya liposome dispersions $O$.

increased more slowly and then levelled off. This plateau value differed between the liposome dispersions, being $\sim 6 \mu \mathrm{g} \beta$-carotene per gram of phospholipid for the MFGM-derived phospholipid and $\sim 3.5 \mu \mathrm{g} \beta$-carotene per gram of phospholipid for the soya-derived phospholipid. ANOVA analysis found that there were significant differences between the entrapment values at $95 \%$.

The data shown in Figure 1 has been used to calculate entrapment efficiencies for $\beta$-carotene (Fig. 2). The differences in efficiency of $\beta$-carotene entrapment were obvious. When very small amounts of $\beta$-carotene were dissolved in chloroform along with the MFGM material, almost all of the $\beta$-carotene became incorporated into the liposome membrane. As more $\beta$-carotene was added, the proportion entrapped decreased. The entrapment efficiency of the soya liposome dispersion was considerably lower than the MFGM liposome dispersion, with efficiency not exceeding 0.5 even at very low $\beta$-carotene concentrations. The entrapment efficiency of the soya liposome dispersion was comparatively less affected by the increasing level of $\beta$-carotene addition.

The steeper slope of the linear portion of the MFGM liposome curve shown in Figure 1 is reflected in the higher entrapment efficiency below $10 \mathrm{mg} \beta$-carotene per gram of phospholipid.

Figure 3 shows the total amount of $\beta$-carotene entrapped per gram of phospholipid using ethanol as the dispersion medium instead of chloroform. It is clear that the use of ethanol significantly reduced the amount of $\beta$-carotene incorporated in the liposome membrane for all samples (compare the $y$-axis on Figs. 1 and 2). The highest amount of $\beta$-carotene added was much lower than for the systems using chloroform as the dispersion medium. This was because during sample preparation there were obviously substantial amounts of aggregated $\beta$-carotene, 


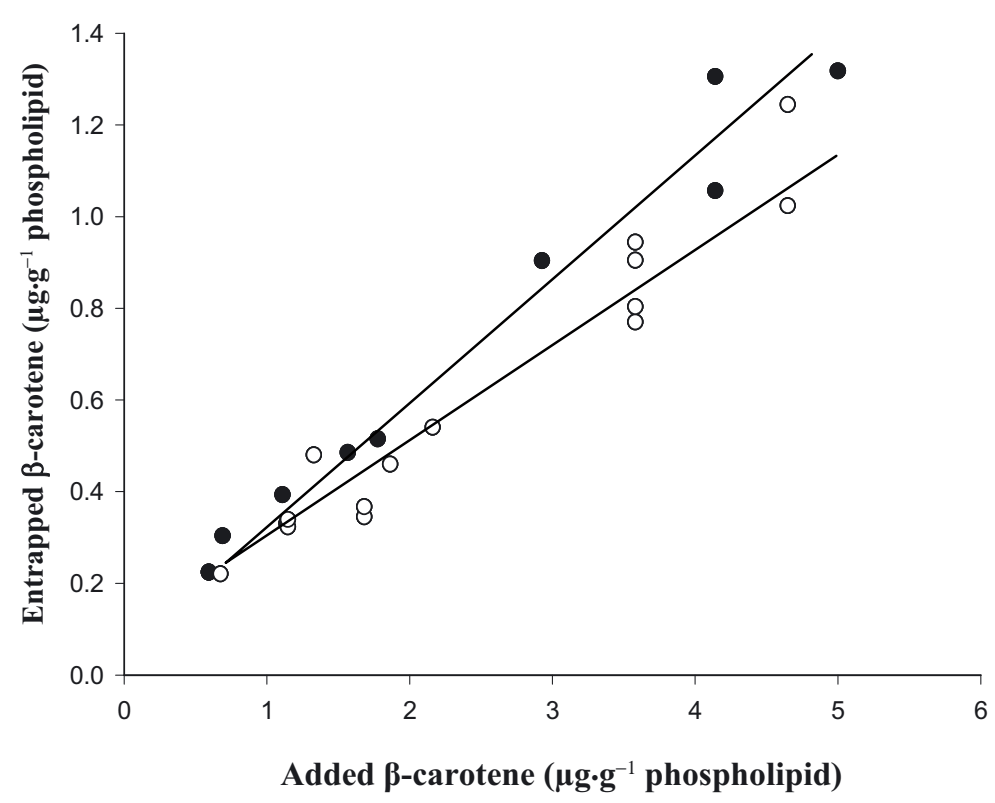

Figure 3. Entrapment of added $\beta$-carotene in liposome membranes using ethanol to disperse the $\beta$-carotene and phospholipid prior to microfluidization. MFGM liposome dispersions $\bullet$, soya liposome dispersions $\bigcirc$.

and the removal of all of the aggregates was blocking the filters and contaminating the gel column. The general shape of the plot shown in Figure 3 is very similar to the portion of Figure 1 between $0-6 \mu \mathrm{g}$ $\beta$-carotene per gram of phospholipid. This suggests that had higher concentrations of $\beta$-carotene been added, the increase in the amount of $\beta$-carotene entrapped using the ethanol as the dispersion medium would be likely to slow down and reach a plateau in the same manner observed when using chloroform.

The entrapment efficiencies for liposome dispersions produced using ethanol are shown in Figure 4. Once again there were similarities with the comparative data obtained using chloroform as the dispersion medium (Fig. 2). The entrapment efficiency was reduced as the amount of $\beta$-carotene added increased, but even at very low levels of $\beta$-carotene the efficiency did not exceed 0.5 for any of the liposome dispersions. While this is significantly lower than the 70-90\% entrapment shown using chloroform, it is encouraging that between $40-50 \%$ of the added $\beta$-carotene can be incorporated in the liposome membrane using a food-safe solvent. However, due to previously mentioned problems regarding the removal of aggregated $\beta$-carotene, the concentrations of $\beta$-carotene used in these experiments are very low compared with those seen in the literature $[2,6,10,11,18,22,24,25]$ or those that would be required for most food applications.

Irrespective of the solvent used, the incorporation of $\beta$-carotene into the phospholipid membrane initially occurs proportionally to the total amount of $\beta$-carotene present in the system, with total $\beta$-carotene entrapped increasing in a linear fashion. Once a certain $\beta$-carotene:phospholipid 


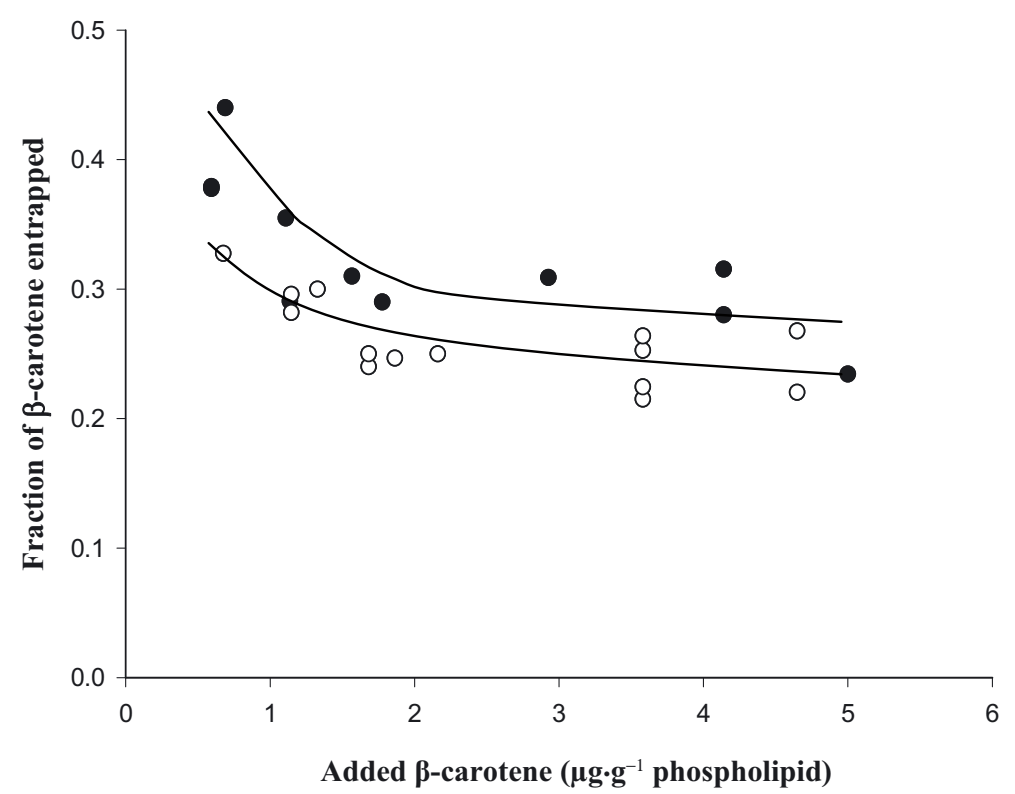

Figure 4. Entrapment efficiency for added $\beta$-carotene in liposome membranes using ethanol to disperse the $\beta$-carotene and phospholipid prior to microfluidization. MFGM liposome dispersions $\bullet$, soya liposome dispersions $\bigcirc$.

ratio is obtained within the membrane, it appears that the membrane becomes saturated and there is only limited increase in entrapped $\beta$-carotene upon further addition of $\beta$-carotene to the system. The MFGM-phospholipid liposome dispersions appeared to reach this point at $\beta$-carotene concentrations of between 6-7 $\mu \mathrm{g}$ per gram of phospholipid, while the soya liposome membranes seemed to become saturated at $\sim 4 \mu \mathrm{g}$ per gram of phospholipid.

The efficiency of entrapment was highest for very low levels of $\beta$-carotene, and reduced linearly as more $\beta$-carotene was added to the system. For liposome dispersions produced using chloroform, there appeared to be a linear relationship between entrapment efficiency and added $\beta$-carotene up to $18 \mu \mathrm{g}$ per gram of phospholipid. The plateau in entrapment efficiency may suggest a partitioning of the $\beta$-carotene between the solvent phase (chloroform or ethanol) and the phospholipid.

In general, the overall trends were comparable between liposome dispersions produced using chloroform and those produced using ethanol, but the entrapment efficiencies were significantly lower in the ethanol-based systems. This was presumably because of the low solubility of $\beta$-carotene in ethanol, less than $0.01 \mathrm{~g} \cdot \mathrm{mL}^{-1}$ compared with $3 \mathrm{~g} \cdot \mathrm{mL}^{-1}$ for chloroform [7]. For the $\beta$-carotene molecules to become incorporated in the phospholipid membrane, they must be solubilised and brought into contact with the phospholipid molecules. If the $\beta$-carotene molecules form aggregates they will not become part of the membrane structure. Shibata et al. [22] found that $\beta$-carotene has a relatively low miscibility in phospholipid membranes, and is inclined to 
form aggregates rather than always becoming incorporated into phospholipid membranes. While the use of chloroform appears to have overcome any miscibility issues in this study, if $\beta$-carotene was poorly soluble in ethanol as well as having low miscibility in the phospholipid membranes there would be strong tendency to form aggregates rather than becoming incorporated into the membrane.

It must be noted that there was some difficulty in dissolving the SigP3644 phospholipid fraction in the solvents. However, increasing the amount of solvent, temperature or length of dispersion time did not have a significant effect on the level of $\beta$-carotene entrapment for this fraction.

The differences in $\beta$-carotene entrapment between the two liposome dispersions are most likely to be due to differences in the composition of the phospholipid fractions. The partitioning of the $\beta$-carotene between the liposome membrane and the solvent phase will be influenced by the hydrophobicity of the membrane interior, which is determined by the fatty acid composition and presence of other hydrophobic molecules. The presence of amphiphilic molecules, including proteins, within the membrane may also affect membrane hydrophobicity.

\subsection{Potassium chromate entrapment}

The absorbance of the eluted fractions for a $5 \%$ phospholipid liposome dispersion with and without entrapped potassium chromate and a solution of free potassium chromate is shown in Figure 5. Similar plots were obtained for all of the phospholipid concentrations for both the MFGMderived and soya-derived liposomes.

The elution profile of the empty liposome dispersion showed a single broad peak starting after $\sim 5$ min (Fig. 5, peak B) and the solution of free potassium chromate solution had a single small peak after $\sim 27 \mathrm{~min}$ (Fig. 5, peak C). These two peaks were used as references to analyze the third dispersion which contained the same concentration of phospholipids as in peak $\mathrm{B}$ and the same potassium chromate concentration as in peak $\mathrm{C}$. This dispersion showed two successive absorption peaks, the first overlapping the peak corresponding to the empty liposomes and the second overlapping the peak from the free potassium chromate.

Peak A was noticeably larger than peak B. As the phospholipid concentration, liposome size and injected volume for these two dispersions was the same, this difference can not be due to an increase in the number or size of liposomes present in the eluted fractions. The most likely reason for the larger peak is the absorbance of entrapped potassium chromate molecules. This hypothesis is further supported by the reduced size of the peak corresponding to the potassium chromate for this dispersion compared to the peak for the same concentration of free potassium chromate.

Based on these observations, we quantified the amount of potassium chromate entrapped by the liposome dispersions. Using a calibration curve for absorbance at known potassium chromate concentrations, if $\mathcal{A}$ represents the area under the curve for the first peak for the liposome dispersion containing potassium chromate and $\mathcal{B}$ the area under the curve for the empty liposome dispersion, then the amount of potassium chromate entrapped $\left(\Delta \mathrm{KCr}_{\mathrm{e}}\right)$ can be calculated from:

$$
\Delta \mathrm{KCr}_{\mathrm{e}}=\mathcal{A}-\mathcal{B} \text {. }
$$

This can then be used to calculate entrapment efficiencies (EE) for the potassium chromate. The EE were calculated as:

$$
\mathrm{EE}(\%)=\frac{\Delta \mathrm{KCr}_{\mathrm{e}}}{\Delta \mathrm{KCr}_{\mathrm{e}}+\mathrm{KCr}_{\mathrm{un}}}
$$

where $\mathrm{KCr}_{\mathrm{un}}$ is the unentrapped potassium chromate which is given by $\mathcal{D}$, the area 


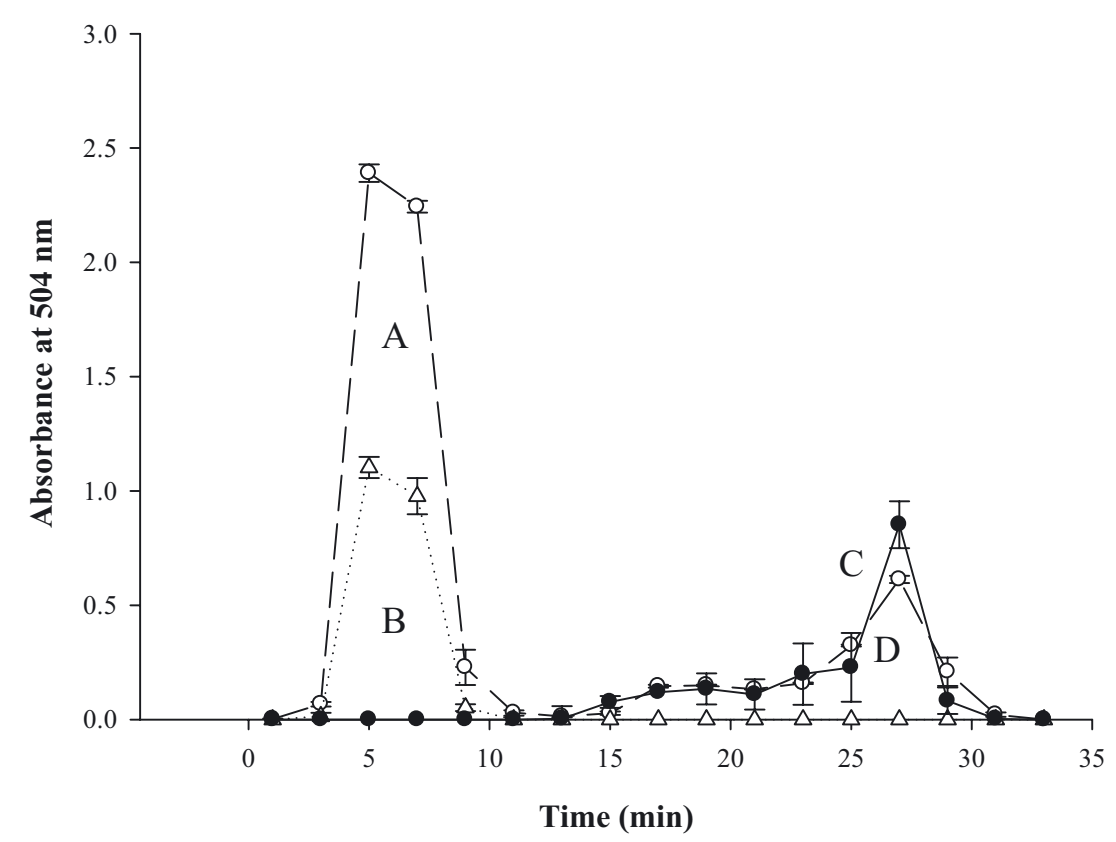

Figure 5. Absorbance of eluted fractions collected over $35 \mathrm{~min}$ for a 5\% MFGM-derived phospholipid dispersion. The plots for three different samples are overlaid: liposome dispersion prepared with entrapped potassium chromate $(O)$, empty liposome dispersion (no potassium chromate) $(\Delta)$ and free potassium chromate (no liposomes) $(\bullet)$. Each data point is the average of two or more replicates and is shown \pm the standard deviation.

under the second peak for the liposome dispersion containing potassium chromate

The EE calculated for different phospholipid concentrations for both MFGMderived and soya-derived phospholipids are shown in Figure 6. As expected, increasing the phospholipids concentration increased the hydrophilic entrapment efficiency.

Higher entrapment efficiencies have been obtained with the MFGM-derived phospholipid dispersions than with the soya dispersions. This is not surprising given that the liposomes formed from the MFGM phospholipids were larger than those produced from the soya phospholipids (Tab. I). The mean diameters were slightly larger for the $10 \%$ phospholipid liposome dispersions than for the 1 or
$5 \%$ dispersions, suggesting that the higher concentration was less sensitive to the shear and turbulence produced during microfluidization. As the EE is usually proportional to the entrapped volume, and the entrapped volume is proportional to the radius to the 3rd power, small differences in diameter can result in significant differences in EE.

Table II compares the ratios of mean diameter and EE for the MFGM and soya liposome dispersions at different phospholipid concentrations. Using the $10 \%$ phospholipid dispersion as an example, it shows that a sphere with a diameter of $98 \mathrm{~nm}$ will only have $77 \%$ the volume of a sphere with a diameter of $110 \mathrm{~nm}$. The differences in measured EE at phospholipid concentrations of 5 and $10 \%$ appear to be at least 


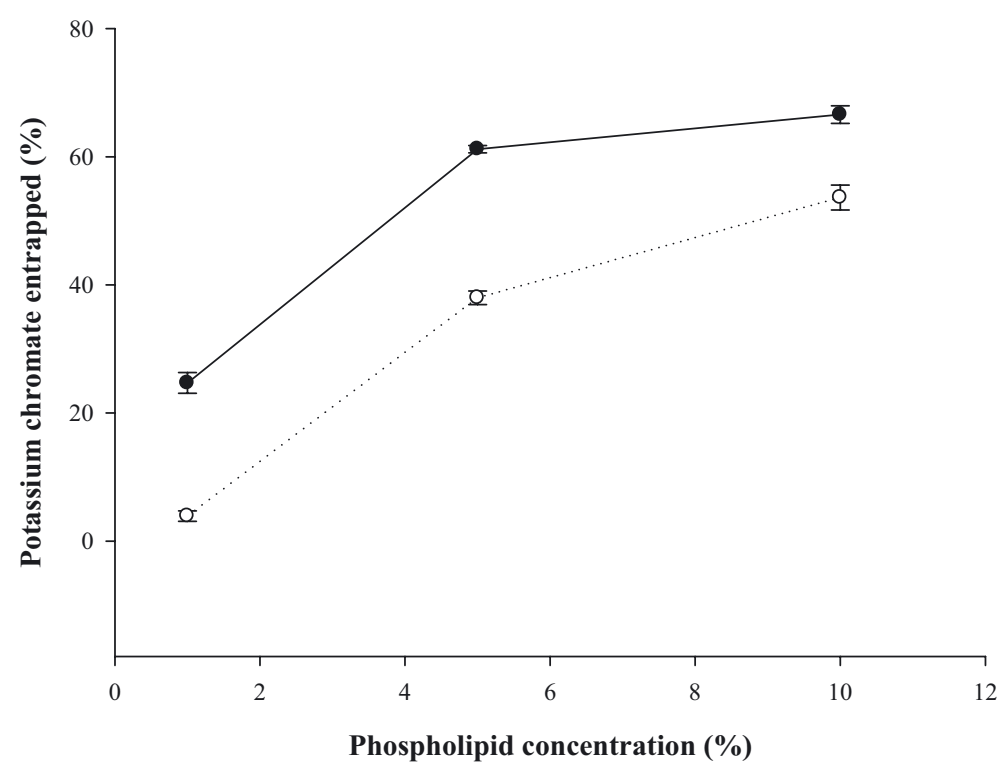

Figure 6. Effect of phospholipid concentration and phospholipid type on the entrapment efficiency for potassium chromate. The types of phospholipid used were derived from MFGM (•) and soya (O) sources. Each data point is the average of five replicates and is shown \pm the standard deviation.

Table II. Effect of phospholipid concentration on the relative volume and entrapment efficiencies of liposomes produced from the MFGM- and soya-derived phospholipids via microfluidization.

\begin{tabular}{lcc}
\hline $\begin{array}{l}\text { Phospholipid } \\
\text { concentration }\end{array}$ & $\begin{array}{l}\text { Ratio of volume of spheres with } \\
\text { diameters corresponding to average } \\
\text { diameter of liposome population } \\
\text { soya liposome/MFGM liposome }\end{array}$ & $\begin{array}{l}\text { Ratio of measured entrapment } \\
\text { efficiencies (EE) }\end{array}$ \\
$(\% \mathrm{w} / \mathrm{w})$ & $0.56 \pm 0.22$ & soya liposome/MFGM liposome \\
\hline 1 & $0.63 \pm 0.13$ & $0.23 \pm 0.05$ \\
5 & $0.77 \pm 0.15$ & $0.64 \pm 0.09$ \\
10 & & $0.79 \pm 0.12$ \\
\hline
\end{tabular}

partly due to differences in the mean diameter of the liposomes. The measured $\mathrm{EE}$ at $1 \%$ phospholipid is smaller than would be expected given the size of the liposomes, but there is large variation in the measured mean diameters for the liposome dispersions at the lower phospholipid concentration. Comparing the lower end of the confidence interval for the ratio of volume of spheres $(0.56-0.22=0.34)$ with the upper end of the ratio of EE values $(0.23+0.05=$ 0.28 ) shows that the difference between these values is not large, and may be due to a slight under-estimation of the variation of the population mean. Overall, the figures are in agreement with those previously reported for liposomes produced via microfluidization [12].

\section{CONCLUSIONS}

These results suggest that liposomes formed from the MFGM-derived phospholipid are able to entrap a higher percentage of the hydrophobic molecule $\beta$-carotene 
than those from soya phospholipid, using either chloroform or ethanol as the dispersion medium. This observation may be due to a more favourable partitioning of the molecule between the aqueous phase and the MFGM-phospholipid membrane. It also seems that the MFGM liposomes entrap a higher percentage of the hydrophilic molecule potassium chromate, but these differences appear to reflect the slightly larger diameter of the liposomes produced from the MFGM material as opposed to be a product of differences in membrane composition.

These results suggest a potential advantage in using MFGM-derived phospholipids, particularly for the production of liposomes for entrapping hydrophobic actives.

Acknowledgements: This work was funded in part by a Bright Future Top Achiever Doctoral scholarship provided by the Tertiary Education Commission (AKT), Massey University and Fonterra Co-operative Ltd.

\section{REFERENCES}

[1] Brandl M., Bachmann D., Drechsler M., Bauer K., Liposome preparation by a new high pressure homogenizer Gaulin Micron LAB 40, Drug Dev. Ind. Pharm. 16 (1990) 2167-2191.

[2] Cantrell A., McGarvey D.J., Truscott T.G., Rancan F., Böhm F., Singlet oxygen quenching by dietary carotenoids in a model membrane environment, Arch. Biochem. Biophys. 412 (2003) 47-54.

[3] Corredig M., Dalgleish D.G., Characterization of the interface of an oil-in-water emulsion stabilized by milk fat globule membrane material, J. Dairy Res. 65 (1998) 465-477.

[4] Crook T., Petrie W., Wells C., Massari D., Effects of phosphatidylserine in Alzheimer's disease, Psychopharmacol. Bull. 28 (1992) 61-66.

[5] Crook T., Tinklenberg J., Yesavage J., Petrie W., Nunzi M., Massari D., Effects of phosphatidylserine in age-associated memory impairment, Neurology 41 (1991) 644-649.
[6] Gabrielska J., Gruszecki W., Zeaxanthin (dihydroxy- $\beta$-carotene) but not $\beta$-carotene rigidifies lipid membranes: a ${ }^{1} \mathrm{H}-\mathrm{NMR}$ study of carotenoid-egg phosphatidylcholine liposomes, Biochim. Biophys. Acta 1285 (1996) 167-174.

[7] Gordon H.T., Bauerfeind J.C., Carotenoids as food colorants, CRC Cr. Rev. Food Sci. 18 (1983) 59-98

[8] Huwiler A., Kolter T., Pfeilschifter J., Sandhoff K., Physiology and pathophysiology of sphingolipid metabolism and signaling, Biochim. Biophys. Acta 1485 (2000) 63-99.

[9] Koopman J., Turkish V., Monto A., Infant formula and gastrointestinal illness, Am. J. Public Health 75 (1985) 477-480.

[10] Lancrajan I., Diehl H., Socaciu C., Engelke M., Zorn-Kruppa M., Carotenoid incorporation into natural membranes from artificial carriers: liposomes and beta-cyclodextrins, Chem. Phys. Lipids 112 (2001) 1-10.

[11] Liebler D., Stratton S., Kaysen K., Antioxidant actions of $\beta$-carotene in liposomal and microsomal membranes: Role of carotenoid-membrane incorporation and $\alpha$-tocopherol, Arch. Biochem. Biophys. 338 (1997) 244-250.

[12] Martin F., Pharmaceutical Manufacturing of Liposomes, in: Tyle P. (Ed.), Specialised Drug Delivery Systems, Marcel Dekker, Inc., New York, 1990, pp. 267-316.

[13] Mayhew E., Lazo R., Vah W.J., King J., Green A.M., Characterization of liposomes prepared using a microfluidiser, Biochim. Biophys. Acta 775 (1984) 169-174.

[14] New R., Introduction, in: New R. (Ed.), Liposomes - a practical approach, IRL Press, Oxford, UK, 1990, pp. 1-32.

[15] Peel M., Liposomes produced by combined homogenization/extrusion, GIT Laboratory Journal 3 (1999) 37-38.

[16] Picon A., Gaya P., Medina M., Nunez M., The effect of liposome encapsulation of chymosin derived by fermentation on Manchego cheese ripening, J. Dairy Sci. 77 (1994) 16-23.

[17] Reineccius G., Liposomes for controlled release in the food industry, in: Risch S., Reineccius G. (Eds.), Encapsulation and controlled release of food ingredients, American Chemical Society, Washington DC, USA, 1995, pp. 113-131. 
[18] Rhim C.-H., Lee K.-E., Yuk H.-G., Lee S.-C., Investigation of the incorporation efficiency of $\beta$-carotene into liposomes, J. Food Sci. Nutr. 5 (2000) 177-178.

[19] Richards M.H., Gardner C.R., Effects of bile salts on the structural integrity of liposomes, Biochim. Biophys. Acta 543 (1978) 508522.

[20] Roesch R.R., Rincon A., Corredig M., Emulsifying properties of fractions prepared from commercial buttermilk by microfiltration, J. Dairy Sci. 87 (2004) 4080-4087.

[21] Sato H., Liu H., Adachi I., Ueno M., Lemaire M., Horikoshi I., Enhancement of the intestinal absorption of a cyclosporine derivative by milk fat globule membrane, Biol. Pharm. Bull. 17 (1994) 1526-1528.

[22] Shibata A., Kiba Y., Akati N., Fukuzawa K., Terada H., Molecular characteristics of astaxanthin and [beta]-carotene in the phospholipid monolayer and their distributions in the phospholipid bilayer, Chem. Phys. Lipids 113 (2001) 11-22.

[23] Škalko N., Bouwstra J., Spies F., Stuart M., Frederik P., Gregoriadis G., Morphological observations on liposomes bearing covalently bound protein: Studies with freezefracture and cryo electron microscopy and small angle X-ray scattering techniques, Biochim. Biophys. Acta 1370 (1998) 151160.

[24] Socaciu C., Bojarski P., Aberle L., Diehl H., Different ways to insert carotenoids into liposomes affect structure and dynamics of the bilayer differently, Biophys. Chem. 99 (2002) 1-5.

[25] Strzalka K., Gruszecki W., Effect of $\beta$-carotene on structural and dynamic properties of model phospholipid membranes. I. An EPR spin label study, Biochim. Biophys. Acta 1194 (1994) 138-142.
[26] Thompson A., Haisman D., Singh H., Stability of liposomes prepared from milk fat globule membrane and soya phospholipids, J. Agr. Food Chem. 54 (2006) 6390-6397.

[27] Thompson A., Hindmarsh J., Haisman D., Rades T., Singh H., Comparison of the structure and properties of liposomes prepared from milk fat globule membrane and soya phospholipids, J. Agr. Food Chem. 54 (2006) 3704-3711.

[28] Thompson A., Mozafari M., Singh H., The properties of liposomes produced from milk fat globule membrane material using different techniques, Le Lait 87 (2007) 349-360.

[29] Thompson A., Singh H., Preparation of liposomes from milk fat globule membrane phospholipids using a microfluidiser, J. Dairy Sci. 89 (2006) 410-419.

[30] Valenti D., De Logu A., Loy G., Sinico C., Bonsignore L., Cottiglia F., Garau D., Fadda A., Liposome-incorporated Santolina insularis essential oil: Preparation, characterization, and in vitro antiviral activity, J. Liposome Res. 11 (2001) 73-90.

[31] Weiner N., Phospholipid liposomes: properties and potential use in flavor encapsulation, in: Flavour Technology, American Chemical Society Symposium Series, 1995, pp. 210 218

[32] Yuasa H., Sekiya M., Ozeki S., Watanabe J., Evaluation of milk fat-globule membrane (MFGM) emulsion for oral administration: absorption of alpha-linolenic acid in rats and the effect of emulsion droplet size., Biol. Pharm. Bull. 17 (1994) 756-758.

[33] Zeisig R., Cämmerer B., Liposomes in the food industry, in: Vilstrup P. (Ed.), Microencapsulation of Food Ingredients, Leatherhead Publishing, London, 2001, pp. 101-119. 